\title{
Philosophiques
}

\section{Un « bilan » vite parcouru}

\section{Maurice Lagueux}

Volume 13, numéro 1, printemps 1986

URI : https://id.erudit.org/iderudit/203308ar

DOI : https://doi.org/10.7202/203308ar

Aller au sommaire du numéro

Éditeur(s)

Société de philosophie du Québec

ISSN

0316-2923 (imprimé)

1492-1391 (numérique)

Découvrir la revue

Citer cet article

Lagueux, M. (1986). Un « bilan » vite parcouru. Philosophiques, 13(1), 153-155.

https://doi.org/10.7202/203308ar

Ce document est protégé par la loi sur le droit d'auteur. L’utilisation des services d'Érudit (y compris la reproduction) est assujettie à sa politique d'utilisation que vous pouvez consulter en ligne.

https://apropos.erudit.org/fr/usagers/politique-dutilisation/
Cet article est diffusé et préservé par Érudit.

Érudit est un consortium interuniversitaire sans but lucratif composé de l’Université de Montréal, l’Université Laval et l’Université du Québec à Montréal. Il a pour mission la promotion et la valorisation de la recherche. https://www.erudit.org/fr/ 
Un «bilan» vite parcouru.

\author{
par Maurice Lagueux
}

À propos de ces années soixante qui nous ont tous marqués au point où leur seule évocation ne laisse personne indifférent, Gérard Raulet est de ceux qui, à n'en pas douter, auraient beaucoup à nous dire. Toutefois le souci bien légitime de faire part aux lecteurs de Philosophiques de ce que cette période toute proche de nous aurait pu lui inspirer n'excuse que partiellement une lecture aussi fantaisiste de mon livre qui, en l'occurrence, servait de prétexte à son intervention en la matière.

Le quiproquo majeur qui explique l'étrange conclusion de son texte tient apparemment à ce qu'il a postulé que j'étais marxiste et supposé en conséquence que, même si j'ai consacré un livre à dénoncer (parfois avec un rien d'ironie que d'autres n'ont pas manqué de me reprocher) nombre d'absurdités associées au marxisme, ce devait être pour inviter tacitement à y croire quand même! À vrai dire, le fait que je n'ai jamais été marxiste ni été tenté de le devenir a bien peu d'importance en tant que tel; ce qui importe cependant, c'est le droit pour qui n'est pas lui-même marxiste, et qui au surcroît est un peu « sceptique», de prendre pour objet et de chercher à expliquer un phénomène aussi insolite et aussi caractéristique des années soixante que la tendance à se dire "néanmoins marxiste», alors omniprésente chez ceux que, sous toutes ses formes conventionnelles, le marxisme avait déçus.

Mais Gérard Raulet a trouvé plus commode de me stigmatiser comme l'homme du «rêve», de la «postulation» et de "l'espérance». Ainsi, au terme de ma critique plutôt sévère de l'argument de Balibar sur la dernière instance, un scrupule de logique m'amenait à concéder - avant de montrer, à la page suivante, que, selon moi, la seule voie valable était ailleurs - qu'on pouvait naturellement postuler ce que manifestement on ne parvenait pas à justifier. C'en était assez pour permettre à Raulet de cueillir là une «clé» qu'il pouvait ensuite promener allègrement dans mon livre pour déverrouiller par son action magique tous les passages qu'il aurait été un peu fastidieux de lire attentivement.

L'espace me manque ici pour souligner tous les aspects du texte de Raulet qui m'ont littéralement laissé pantois, que ce soit à propos de "l'ambiguïté» qu'entretiendrait ma condamnation, que je croyais sans appel, de tout recours pour fin épistémologique au «point de vue du prolétariat», que ce soit à propos du présumé dessein de mon livre qui serait d'«esquisser une histoire idéologique du Québec» (sic) ou même à propos de cette «érudition» qu'avec une amabilité vraiment excessive Raulet, à deux reprises, prétend déceler dans mon travail.

Je lui concède volontiers une seule chose, c'est que le titre de mon livre pouvait prêter à équivoque: il ne porte pas sur tout ce qui a pu être associé 
au marxisme au cours des années soixante. Aussi, à défaut d'avoir trouvé la façon d'exprimer dans un titre qui sonne agréablement aux oreilles d'un éditeur le programme précis de mon ouvrage, j'y ai ajouté un avant-propos de moins de six pages que, faute de faire plus, Gérard Raulet aurait pu, au moins, lire avec attention. L'eût-il fait, il y aurait trouvé (p. 12-13) la définition de la «pensée critique» qu'il se plaint d'avoir cherché en vain "dans l'ouvrage». Par la même occasion, il aurait appris que le livre prétendait porter avant tout sur un choix d'arguments représentatifs de cette époque. Surtout - mais il aurait dû alors renoncer à le ridiculiser en le présentant comme un «bilan du marxisme» - il ne se serait pas contenté de glaner un seul mot d'une formule quand même un peu moins prétentieuse : «[...] une sorte de bilan provisoire ou mieux un retour lui-même critique sur une période encore très proche de nous [...]» (p. 13, soulignés ajoutés). Enfin, il aurait été renvoyé à une note soulignant que mon texte sur l'bomo sobrius est celui qui remonte le plus loin dans le temps (1974), et aurait pu s'épargner ainsi de rater d'aussi loin la marque en lançant à tout hasard l'hypothèse voulant que je l'aie rédigé à seule fin de donner plus d' «actualité » à mon livre, tout ça, parce que j'y emploie (une seule fois et sur un ton amusé) l'épithète "post-industriel» qui constituerait pour lui le fin mot d'une analyse des années '70.

Manifestement, Raulet est déçu de voir que je me situe aussi mal dans un cadre approprié à l'examen des débats qui l'intéressent. Fallait-il en conclure que je me refuse à toute position personnelle? J'avoue m'y perdre un peu dans la comptabilité à laquelle il recourt sur le caractère secondaire, tertiaire ou secondaire au carré de la littérature sur ces questions. Pour ma part, sans revendiquer plus que ça de faire ceuvre de littérature «primaire», j'ai voulu prendre pour objet un phénomène culturel récent que, à ma connaissance, personne n'avait encore examiné en tant que tel. Et je ne vois aucun paradoxe à ce que, de ce point de vue, je me sois intéressé à l'épisode althussérien bien que, je dois le reconnaître, je serait peu enclin à soutenir qu'il n'a rien de « superflu».

Car dire que la pensée critique avait «le vent dans les voiles» au cours des années ' 60 , ce n'est pas se montrer bêtement « optimiste», c'est dire qu'il fallait bien que la gauche jouisse à l'époque d'une faveur exceptionnelle pour que des énormités comme celles que l'on rencontre si abondamment chez Althusser et ses disciples aient pu susciter un peu partout, en Europe et en Amérique, un enthousiasme qu'on parvient mal à s'expliquer depuis que ce vent est tombé. Au risque de brusquer quelques idées reçues sur le sujet ou - pour employer le langage volontiers pictural de Gérard Raulet - au risque de "rendre tout flou», j'ai cru que ce phénomène insolite méritait bien d'être examiné pour lui-même. Raulet, pour sa part, estime qu'un livre qui se consacre à une tâche aussi peu inspirée - s'il ne peut se targuer d'avoir «apporté sa pierre au redéploiement de la "pensée critique"» et s'il ne débouche même pas sur une « relecture des textes méthodologiques de Marx» - «n'a pas de raison d'être» et, en conséquence, ne mérite pas d'être « publié ». À plus forte raison, et cette conclusion, semble-t-il ne lui a 
pas échappé, ne mérite-t-il pas d'être lu. Tout au plus mérite-t-il de faire l'objet d'une étude critique.

Département de philosopbie

Université de Montréal

* 